\title{
Les forêts lorraines dans la tempête du 26 décembre 1999 : premier bilan
}

The Lorrain forests in the storm of the 26th December 1999 : first assessment Die Wälder Lothringens im Sturm des 26. Dezember 1999 : Erste Bilanz

\section{Robin Degron}

\section{(2) OpenEdition}

\section{Journals}

Édition électronique

URL : http://journals.openedition.org/rge/4107

DOI : $10.4000 /$ rge.4107

ISSN : $2108-6478$

Éditeur

Association des géographes de l'Est

Édition imprimée

Date de publication : 1 juin 2000

ISSN : 0035-3213

\section{Référence électronique}

Robin Degron, «Les forêts lorraines dans la tempête du 26 décembre 1999 : premier bilan », Revue Géographique de l'Est [En ligne], vol. 40 / 3 | 2000, mis en ligne le 02 août 2013, consulté le 08 septembre 2020. URL : http://journals.openedition.org/rge/4107 ; DOI : https://doi.org/10.4000/rge. 4107

Ce document a été généré automatiquement le 8 septembre 2020

Tous droits réservés 


\section{Les forêts lorraines dans la tempête du 26 décembre 1999 : premier bilan}

The Lorrain forests in the storm of the 26th December 1999 : first assessment

Die Wälder Lothringens im Sturm des 26. Dezember 1999 : Erste Bilanz

Robin Degron

\section{Introduction}

1 Alors que l'on redoutait l'effondrement des tours informatiques, ce sont les forêts, futaies cathédrales ou modestes chapelles de taillis-sous-futaie, qui ont vacillé et parfois chu sous les assauts du vent de la fin 1999. Les tempêtes des 26 et 28 décembre derniers laisseront sans doute de profondes marques au sein des peuplements et dans l'esprit des forestiers français. Même dans les systèmes tempérés, l'influence des aléas naturels parait toujours grande (B. Ledoux, 1995).

2 Si l'incertitude reste encore importante autour des premières données disponibles, il semble cependant aujourd'hui possible d'établir un bilan, provisoire, de l'impact de la tempête du 26 décembre 1999 sur les forêts lorraines. En dépassant les effets d'annonce médiatique, la simple collecte des estimations dispersées au sein des administrations compétentes permet d'obtenir une vue de synthèse pour le géographe et, au-delà, pour le citoyen. Quel tableau dresser d'une des principales régions forestières françaises aujourd'hui ? Quelles orientations nouvelles donner à la gestion des bois? La perspective de développement durable du patrimoine forestier régional est-elle brisée ?

Partant d'une analyse critique des sources disponibles, nous verrons que la Lorraine a été particulièrement touchée et que de fortes disparités apparaissent dans la région, en fonction des départements et des propriétaires. Nous nous interrogerons enfin sur le devenir de la gestion sylvicole et sur les pistes de réflexion ouvertes au lendemain de la tempête. 


\section{Un bilan difficile à établir}

Encore très imprécise, la mesure des conséquences forestières de la tempête porte pour l'instant sur des facteurs de production simples. De par l'étendue des massifs touchés et la complexité des géosystèmes forestiers, un bilan précis des dégâts, tant sur le plan dendrométrique, écologique, économique et social ne peut être envisagé que sur le moyen-long terme.

\section{A. Des données encore très approximatives et partielles}

\section{Des estimations en volume et en surface très imprécises}

5 Bien que la forêt française soit réputée gérée de façon multifonctionnelle, assurant à la fois un objectif de production de bois, d'accueil du public et de préservation de la biodiversité, la dimension économique demeure essentielle, en particulier en Lorraine (J.P. Husson, 1991) : elle est source de richesse, créatrice d'emplois tant dans le domaine de la gestion et de l'exploitation forestière que dans celui de l'industrie. Ainsi, après la tempête, l'attention des forestiers s'est d'abord portée sur des facteurs de production simples : les volumes abattus et les surfaces touchées.

Etant donné qu'il était pratiquement impossible de cuber les arbres à terre au lendemain de la tempête, les premières estimations en volume de chablis sont des évaluations «à dire d'expert » appuyées sur des observations de terrain. Le travail a été réalisé sur l'ensemble du territoire national. En se basant sur la connaissance des volumes sur pied dans les parcelles, connus avant la tempête grâce aux documents d'aménagement, les sylviculteurs ont pu approcher l'importance des chablis. La méthode reste évidemment très imprécise; ses résultats sont très approximatifs, en particulier pour la forêt privée où les documents de gestion sont beaucoup plus succincts qu'en forêt publique. A travers l'expérience acquise en Auvergne et en Bretagne après les importantes tempêtes de 1982 et 1987, le Ministère de l'Agriculture estime qu'une imprécision d'environ $30 \%$ peut entacher les volumes annoncés.

Différentes méthodes ont été employées pour l'estimation des surfaces de forêts touchées : elles varient selon que l'on considère l'échelle nationale ou locale. A l'échelle nationale, une première estimation a pu être obtenue grâce à l'analyse des placettes du réseau de suivi des dommages forestiers géré par le Département Santé des Forêts (DSF) du Ministère de l'Agriculture (V. Badeau, 1998) : réparties sur l'ensemble du territoire de façon à représenter la diversité des écosystèmes forestiers, les placettes offrent un échantillonnage précieux pour le suivi de l'état sanitaire des forêts ; leur visite après la tempête a permis de se faire une première idée globale de l'ampleur des chablis (Ministère de l'Agriculture et de la Pêche, 2000). L'agrégation des estimations en surface issues des visites de terrain ou des observations aériennes permet également d'obtenir une vue de synthèse nationale. Le problème des seuils de sensibilité s'est évidemment posé : $90 \%, 80 \%$ voire $50 \%$ de tiges tombées sont autant de seuils possibles pour classer un peuplement dans la catégorie de ceux fortement touchés. Globalement, les seuils $90 \%$ et $80 \%$ ont été privilégiés. L'écart de 10 points qui les sépare est peu significatif: les deux seuils renvoient en effet à une réalité très comparable de parcelle dévastée où émergent éventuellement quelques semenciers, incapables d'assurer à eux seuls la régénération naturelle du peuplement préexistant. 
Concernant les parcelles peu touchées, les pourcentages de tiges tombées servant de référence oscillent entre $10 \%$ et $30 \%$. Là encore, les écarts sont à relativiser : il s'agit de peuplement avec des chablis épars, dont l'évolution sylvicole ne devrait pas être trop remise en cause par la tempête. Au-delà du problème des seuils, se pose aussi la question des méthodes d'inventaire utilisées : à l'échelle locale, elles sont variées, plus ou moins précises. La Lorraine et ses départements offrent un bon exemple des différentes approches retenues, en particulier dans les forêts publiques. Ainsi dans les Vosges, une campagne de photographie aérienne a rapidement été lancée par l'office National des Forêts (ONF) : l'interprétation des clichés, le report sur carte puis la transposition sous système d'information géographique offrent ainsi une base de travail solide. En Meuse, un survol des massifs par hélicoptère a permis d'apprécier globalement l'étendue des dégâts avant de les reporter sur les plans d'aménagement ; un inventaire réalisé par les forestiers de terrain a complété l'information. En Meurtheet-Moselle et en Moselle, seule l'enquête parcellaire a été effectuée. Dans les forêts privées de Lorraine, les techniciens du Centre Régional de la Propriété Privée (CRPF) ont pratiqué une reconnaissance par voie terrestre afin d'établir les premières estimations.

\section{Une vue encore partielle des impacts de la tempête}

8 En marge de l'évaluation dendrométrique et spatiale des conséquences de la tempête, l'analyse des effets écologiques, sociaux-économiques voire psychologiques du phénomène reste largement à explorer. L'évolution des populations faunistiques et floristiques liée à la transformation du milieu, l'influence économique du phénomène sur la filière forêt-bois, la mesure du linéaire de sentier de randonnée devenu impraticable ou l'impact mental des destructions de la tempête sur les forestiers sont quelques unes des dimensions encore mal connues sur le plan national ou régional. Au niveau départemental, des campagnes d'inventaire spécifique ont pu être lancées. C'est le cas notamment dans les Vosges pour le recensement des sentiers de randonnées barrés par les chablis. L'importance de l'activité touristique dans ce département justifiait la réalisation rapide d'un tel état des lieux ${ }^{1}$.

\section{B. Une révision très attendue de l'inventaire forestier national}

\section{Les problèmes méthodologiques rencontrés}

9 Au-delà des estimations plus ou moins précises réalisées par les services forestiers gestionnaires, publiques ou privées, l'Inventaire Forestier National (IFN) a évidemment été mobilisé afin d'établir un bilan précis, méthodologiquement cadré au niveau national, décliné dans chaque département (IFN, 2000).

10 Compte tenu de l'immensité de la tâche à accomplir, l'idée d'une couverture satellitaire du territoire a été testée dans un premier temps. A partir d'une interprétation d'images LANDSAT, SPOT ou radar, il s'agissait de cartographier rapidement les zones touchées et d'évaluer leur importance. Trois massifs échantillons ont été retenus afin d'essayer la méthode: le massif des Landes de Gascogne dans son ensemble, les forêts de Fontainebleau et d'Ussel. Avec des peuplements relativement homogènes, à base essentiellement de pins maritimes, et du fait d'une topographie quasiment plane, le massif des Landes s'est bien prêté à l'expérimentation : l'IFN dispose aujourd'hui de la 
carte des dégâts pour les départements des Landes, de la Gironde et du Lot-et-Garonne. La méthode par analyse d'image satellitaire n'a pas pu en revanche être validée pour des forêts plus biodiversifiées que la pineraie maritime, sises sur des modelés complexes (F. Wencélius, 2000).

\section{Un bilan précis d'ici fin 2001}

11 Ainsi, pour évaluer de façon générale les effets de la tempête dans les forêts françaises, une campagne de reconnaissance aérienne classique suivie de photo-interprétation au $1 / 30000$ e est nécessaire. Le travail à réaliser paraît considérable. Même si les territoires a priori les moins touchés sont exclus de la couverture, comme c'est par exemple le cas dans le nord de la Lorraine qui n'a pas été parcouru par la tempête du 26 décembre, on ne peut guère espérer connaitre les résultats de l'IFN avant le deuxième semestre 2001.

12 A cette période devrait être connue la répartition des chablis selon quatre classes de dégâts : $0-10 \% ; 10-50 \% ; 50-90 \%$ et $90-100 \%$ de tiges abattues. Après ce premier inventaire global, il restera évidemment à revoir les différents inventaires départementaux : alors que le programme d'avant tempête de l'IFN prévoyait un nouvel inventaire des forêts lorraines pour 2005, il faudra sans doute raccourcir les délais (F. Wencélius, 2000. Op. cit.).

\section{Des forêts lorraines particulièrement touchées}

Deuxième région française par la surface boisée, dans l'axe de la tempête du 26 décembre 1999, la Lorraine a été particulièrement touchée, dans l'absolu comme sur le plan relatif. Entre les départements et selon les types propriétés, des différences de sensibilité apparaissent.

\section{A. La forêt lorraine première victime d'une tempête exceptionnelle}

\section{Un phénomène d'une ampleur exceptionnelle}

14 Les tempêtes de la fin décembre 1999 ont jeté à terre environ $138 \mathrm{M}$ de m3 de bois chablis. Ramené au capital sur pied des forêts françaises, estimé au début des années 1990 par l'IFN à près de 2 milliards de m3, 7 \% de la ressource du pays ont été affectés, soit deux fois l'accroissement annuel moyen du patri- moine national. Bien qu'il soit entaché d'une forte incertitude (supra), le volume annoncé par le Ministère de l'Agriculture correspond à un phénomène d'ampleur européenne, sans précédent à l'échelle de la période contemporaine (D. Doll, 1991). La figure 1 représente pour la France l'évolution depuis un siècle des volumes de bois chablis liés à de grands événements climatiques, au moins de portée départementale. Le caractère exceptionnel des tempêtes de fin décembre apparaît clairement. En 1982, dans le Massif central, $12 \mathrm{M}$ m3 seulement étaient tombés : il s'agissait du plus grand coup de chablis enregistré au XXe siècle. Un facteur dix sépare la situation actuelle de celle d'il y a dix-huit ans (figure 1). 
Fig. 1 : Volumes de bois chablis tombés en France depuis un siècle sous l'effet de grandes tempêtes (en M m3).

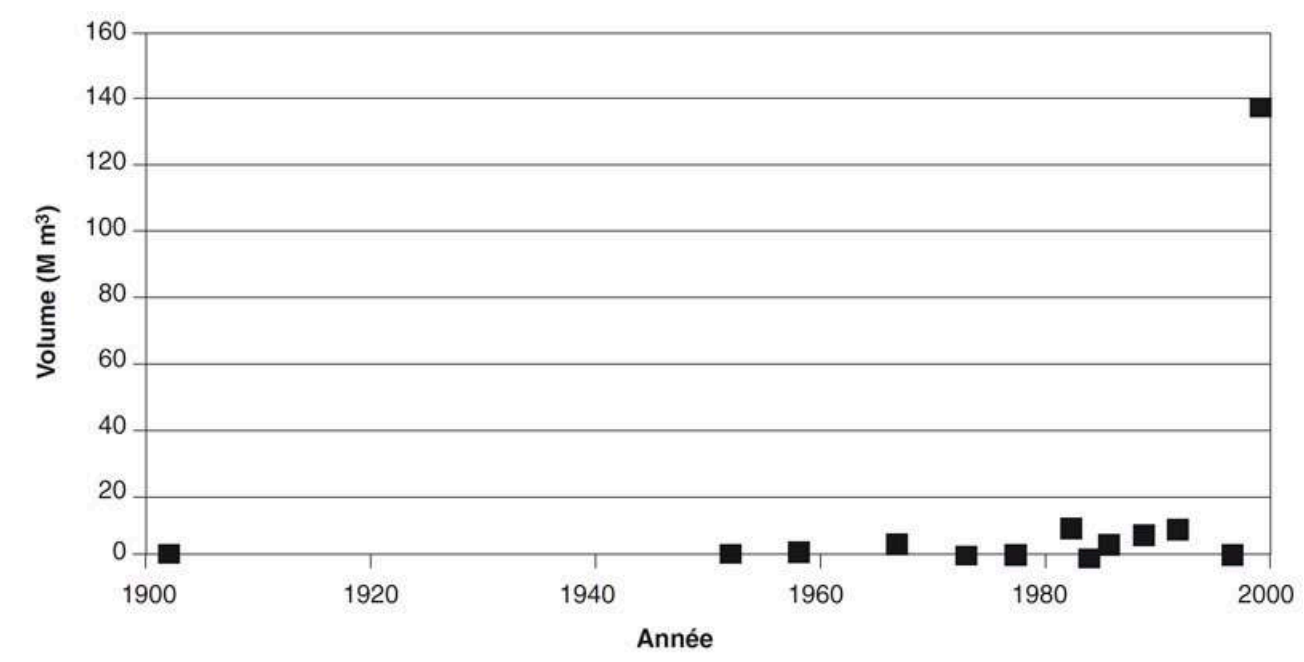

15 Au-delà des estimations en volume, le Ministère de l'agriculture évalue à environ 500 000 ha la surface de forêt fortement touchée, c'est-à-dire quasiment détruite, avec plus de $80 \%$ des tiges tombées. D'après cette estimation officielle, ce sont ainsi 3,6 \% des 14 $\mathrm{M}$ ha de la forêt française qui sont fortement atteints. L'analyse des placettes du réseau européen de suivi des dommages forestiers permet d'affiner un peu le bilan, en particulier concernant les surfaces moyennement touchées (Ministère de l'Agriculture et de la Pêche, 2000. Op. cit.). Sur les 544 placettes du dispositif, 74 \% sont indemnes; $19 \%$ sont moyennement touchées avec moins de $50 \%$ de tiges tombées; $7 \%$ sont fortement atteintes avec plus de $50 \%$ d'arbres chablis. Bien que le seuil de référence des peuplements fortement atteints soit plus bas que celui classiquement retenu par les forestiers de terrain, on peut remarquer que les deux estimations sont compatibles entre elles.

\section{La forêt lorraine très durement touchée}

En Lorraine, on estime que 29,6 M m3 étaient à terre au lendemain du 26 décembre 1999. A elle seule, la région représente $21 \%$ du volume de chablis national. L'Aquitaine avec $20 \%$, le Limousin avec $12 \%$, la Champagne-Ardenne avec $10 \%$ et le PoitouCharentes avec $9 \%$ suivent parmi les régions les plus touchées. Bien sûr, la part de la Lorraine dans le bilan national s'explique en partie par la richesse spécifique de ses forêts avant la tempête. Avec environ $178 \mathrm{M} \mathrm{m} 3$ sur pied au début des années 1990, la Lorraine concentre en effet à elle seule $9 \%$ du stock forestier français: fortement boisée, dotée de peuplements relativement enrichis, la région a beaucoup à perdre ${ }^{2}$. Afin de bien saisir l'intensité des dégâts lorrains, il faut rapporter les pertes subies au volume régional sur pied d'avant tempête : $17 \%$ de ce stock ont été mis à terre, soit quatre fois l'accroissement annuel moyen du massif lorrain. Sur le plan relatif, on peut ainsi considérer que la région a été deux fois plus touchée que la moyenne nationale.

D'après les estimations de l'ONF et du CRPF, 363200 ha de forêt ont été plus ou moins touchés par la tempête, ce qui laisse seulement $57 \%$ des peuplements indemnes. Dans les forêts publiques, sur les 322200 ha concernés, 49200 ha sont détruits à plus de $80 \%$ en nombre de tiges tombées. En forêt privée, on sait juste qu'environ 41000 ha de bois ont été touchés. En se basant uniquement sur les données fournies par l'ONF, on voit 
que $6 \%$ au moins des forêts lorraines sont totalement détruites. Relativement au niveau national où seulement $3,6 \%$ des peuplements sont fortement touchés, la situation lorraine paraît très sensible. Les résultats de l'analyse en surface rejoignent ceux de l'analyse en volume.

\section{B. De fortes disparités au sein de la région}

\section{La comparaison quantitative des situations départementales et entre propriétaires}

Les quantités de chablis et l'impact relatif de la tempête sont assez différents d'un département lorrain à l'autre et entre les différents types de propriété. Le tableau 1 donne le cubage de bois tombé en fonction du département et du propriétaire ainsi que le volume de chablis rapporté au volume sur pied d'avant tempête (tableau 1).

Tableau 1 : Les chablis de la fin 1999 en Lorraine par département et type de propriété (en M m3 et en \% du volume sur pied d'avant tempête)

\begin{tabular}{|l|c|c|c|c|}
\hline & Forêt domaniale & Bois communaux & Forêt privée & Total \\
\hline Meurthe-et-Moselle & $2,4(33 \%)$ & $4,2(30 \%)$ & $1,8(19 \%)$ & $8,4(27 \%)$ \\
\hline Meuse & $1,9(22 \%)$ & $3,3(21 \%)$ & $1,5(13 \%)$ & $6,7(18 \%)$ \\
\hline Moselle & $1,5(8 \%)$ & $0,7(7 \%)$ & $1,4(14 \%)$ & $3,6(9 \%)$ \\
\hline Vosges & $3,2(21 \%)$ & $5,3(16 \%)$ & $2,4(10 \%)$ & $10,9(15 \%)$ \\
\hline Lorraine & $9,0(18 \%)$ & $13,5(18 \%)$ & $7,1(13 \%)$ & $29,6(17 \%)$ \\
\hline
\end{tabular}

Avec 10,9 M m3 à terre, le département des Vosges parait le plus touché en Lorraine. Il est également celui où le taux de boisement et le volume sur pied sont les plus importants ${ }^{3}$. Avec $8,4 \mathrm{M} \mathrm{m} 3$ de chablis, soit $27 \%$ du stock forestier d'avant tempête, c'est en fait le département de Meurthe-et-Moselle qui est relativement le plus touché, devant la Meuse avec $18 \%$, les Vosges avec $15 \%$ et la Moselle avec $9 \%$. C'est l'équivalent de 8 fois l'accroissement annuel moyen qui est tombé en Meurthe-etMoselle, contre seulement 4 fois dans les Vosges.

La comparaison entre type de propriétaire montre quant à elle une sensibilité particulière des forêts publiques par rapport aux forêts privées. Si la part du volume sur pied à terre est quasiment identique dans les forêts domaniales et les bois communaux, un écart de 14 à 6 points apparaît en revanche entre les forêts publiques et privées, en général moins atteintes, sauf en Moselle. Dans ce département, les forêts privées ont en effet perdu 1,4 M m3, pratiquement autant qu'en forêt domaniale alors que les propriétés de l'Etat prédominent ici largement ${ }^{4}$.

21 L'analyse des surfaces touchées conforte et complète les résultats de celle des volumes tombés. Pour les quatre départements lorrains et en ce qui concerne les forêts publiques seulement, le tableau 2 donne les surfaces dévastées, là où 80 à $90 \%$ des tiges sont tombées, ainsi que les surfaces moyennement ou faible- ment touchées (tableau 2). 
Tableau 2 : Les surfaces de forêt publique lorraine touchées par la tempête de la fin 1999 (en ha et en pourcentage de la surface forestière publique totale)

\begin{tabular}{|l|c|c|c|}
\hline & Forêts dévastées & Autres forêts touchées & Surface forestière totale \\
\hline Meurthe-et-Moselle & $13400(12 \%)$ & $47200(43 \%)$ & 110200 \\
\hline Meuse & $13800(9 \%)$ & $46700(31 \%)$ & 151900 \\
\hline Moselle & $4000(3 \%)$ & $9900(8 \%)$ & 121300 \\
\hline Vosges & $17900(10 \%)$ & $169200(90 \%)$ & 187100 \\
\hline Lorraine & $49200(9 \%)$ & $272800(48 \%)$ & 568300 \\
\hline
\end{tabular}

22 Comme lors de l'analyse des dégâts en volume, on retrouve la particulière sensibilité des forêts de Meurthe-et-Moselle où $12 \%$ des massifs publics sont dévastés. A travers l'approche par surface, l'impact de la tempête dans les Vosges apparaît en revanche plus nettement que ne le laissait envisager l'analyse par volume. Toutes les forêts publiques vosgiennes ont été touchées. En plein dans l'axe de la tempête, les massifs forestiers de ce département sont tous plus ou moins atteints: $10 \%$ des forêts publiques sont dévastées ; pour le reste, les dégâts paraissent variables et diffus.

\section{Une approche qualitative de la variabilité des dégâts}

En attendant un inventaire et une cartographie précise des dégâts causés par la tempête, les observations de terrain réalisées par l'office National des Forêts permettent d'établir une carte provisoire des zones touchées. Peu ou pas atteint, le nord de la Lorraine, au Nord d'une ligne brisée Verdun/Metz/Sarrebourg, s'oppose au sud. D'Ouest en Est, les secteurs des Côtes d'Argonne, de Meuse, de Moselle et des Basses-Vosges gréseuses sont particulièrement affectés (figure 2). 
Fig. 2 : Zones forestières touchées par la tempête du 26/12/1999 en Lorraine

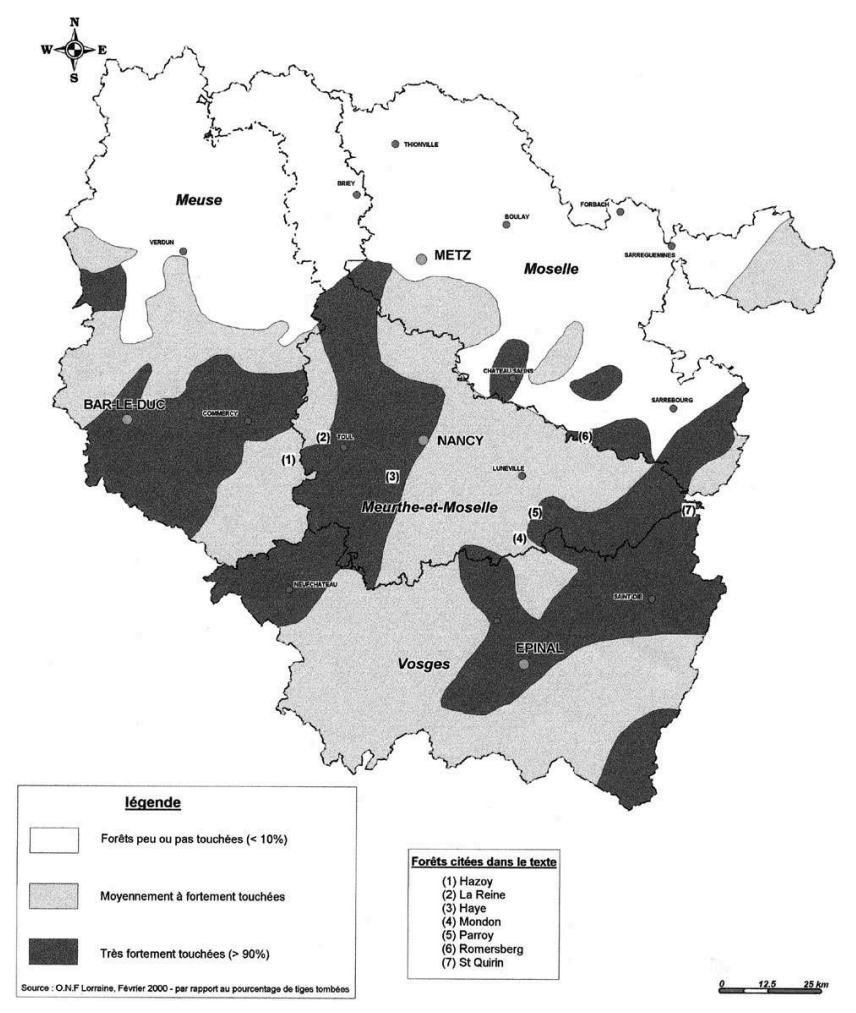

Les sorties de terrain que nous avons réalisées dans des massifs échantillons des Côtes de Meuse (forêt du Hazoy), de la Woëvre (forêt de la Reine), des Côtes de Moselle (forêt de Haye), du Plateau Lorrain (forêt de Parroy, de Mondon et du Romersberg) et des Basses-Vosges (forêt de Saint Quirin), permettent d'enrichir un peu l'analyse biogéographique de la sensibilité différentielle des peuplements à la tempête. Si les forêts de revers de côtes paraissent en effet particulièrement touchées, par exemple en forêt de Haye ou du Hazoy, elles correspondent également, dans une très large mesure, à des hêtraies. Le Hêtre, naturellement dominant sur les substrats calcaires ou gaizeux, présente un enracinement traçant, superficiel, qui le rend particulièrement sensible au coup de vent. Le Chêne, dont l'enracinement est plus profond que celui du Hêtre, prédomine en Woëvre et sur le Plateau Lorrain. Assis sur des sols souvent argileux ou argilo-limoneux, relativement lourds et compacts, il parait relativement stable: les forêts de la Reine et de Parroy ont été ainsi relativement peu touchées. Là où le sol est le plus léger en revanche, par exemple en forêt de Mondon, sur un substrat de type alluvionnaire, d'importants chablis peuvent être constatés. En montagne, les peuplements résineux prédominent. L'enracinement superficiel couplé à une fragilité biomécanique naturelle, liée à la faible densité de leur bois, concourt à la formation d'un paysage de chablis complexe où se mêlent arbres déracinés, c'est-à-dire des chablis au sens strict, mais aussi des volis, c'est-à-dire des arbres cassés au niveau du tronc. S'il paraît aujourd'hui bien difficile de pointer le ou les éléments déterminants de la sensibilité des peuplements au coup de vent, il convient en tous cas de souligner la complexité des facteurs à prendre en compte et de leurs interactions. Concernant la question éminemment sensible pour les aménageurs forestiers de l'influence des traitements sylvicoles pratiqués sur la plus ou moins grande fragilité aux coups de vent des peuplements, il convient là aussi d'être prudent. Entre la forêt de Haye, où prédomine la futaie régulière, et la forêt du Hazoy avec son Taillis-sous-Futaie enrichi, 
le même type de paysage forestier dévasté s'expose. Dans les deux cas, la hêtraie domine.

\section{Une gestion forestière remise en cause?}

Si le bilan des effets de la tempête demeure évidemment à affiner, la situation actuelle appelle d'ores et déjà un certain nombre d'interrogations. Le problème de la reconstitution à brève échéance des forêts plus ou moins touchées ne doit pas faire oublier la dimension essentielle du temps long.

\section{A. Une interrogation sur le court et le long terme}

\section{Quel mode de reconstitution des peuplements?}

Après la mise en sécurité des forêts, l'exploitation, l'éventuel stockage et la commercialisation des bois chablis, un énorme chantier de reconstitution s'ouvre. Tant pour des raisons économiques, qu'écologiques, il semble que les aménageurs veuillent opter pour des interventions minimales, en forêt publique comme en forêt privée : les gestionnaires s'accordent pour laisser venir le recru ligneux et, secondairement, pour reboiser les zones les plus atteintes avec des techniques de plantation à faibles espacements. Les orientations générales données par l'ONF et les réflexions au sortir de tournée de terrain sur le thème de l'après tempête vont dans le même sens ${ }^{5}$.

\section{La gestion forestière durable à l'épreuve de la tempête}

27 La gestion forestière des forêts lorraines, composées d'essence à croissance lente comme le Chêne, le Sapin ou le Hêtre, s'inscrit nécessairement dans une perspective de gestion durable, à l'échelle d'un ou deux siècles. La réflexion à cette échelle permet de relativiser l'impact de la tempête sans pour autant amoindrir les difficultés aujourd'hui rencontrées par les propriétaires forestiers et leurs gestionnaires.

La tempête peut être considérée comme un facteur a priori naturel de rajeunissement des écosystèmes forestiers (J.C. Rameau, 2000). Dans la mesure où la priorité est donnée à la régénération naturelle des peuplements, une biodiversité accrue peut être attendue à terme dans les forêts lorraines. Le problème vient du fait que ce rajeunissement naturel des massifs lorrains vient en résonance avec un phénomène cette fois-ci anthropique d'intensification de la gestion au cours des dernières décennies. Dans les forêts de plaine en particulier, la conversion des Taillis-sous-Futaie en futaie régulière, engagée à partir du Second Empire, ne s'est pas faite de façon régulière, construisant une forêt instable, composée de peuplements dont les classes d'âge sont déséquilibrées. Après un rapide démarrage des conversions durant la deuxième moitié du XIXe siècle, la gestion forestière a été ralentie pendant l'Entre-deux-guerres. Au début des Trente Glorieuses, l'activité sylvicole est remontée en puissance. Les vieux peuplements ont été intensivement renouvelés par plantation ou par voie naturelle. Au début des années 1990, on estime ainsi qu'environ le tiers des forêts du Grand Plateau Lorrain étaient âgées de moins de trente ans (R. Degron, 1999). Un récent rapport de l'IFN confirme d'ailleurs ce phénomène de rajeunissement sylvicole et de décapitalisation dans les hêtraies-chênaies pour l'ensemble du secteur Nord-Est (IFN, 1999). Ainsi, la tempête du 26 décembre 1999 ne fait que renforcer, brutalement, une tendance déjà forte au sein 
des massifs lorrains. Les déséquilibres constatés sur le long terme de la gestion forestière continuent de se creuser : ils tendent à remettre en cause la possibilité d'application du principe de développement durable des géosystèmes forestiers régionaux. Très concrètement, du strict point de vue économique, essentiel dans les forêts lorraines, on peut craindre que l'excès de bois généré conjoncturellement par la tempête n'ouvre une période de relative pénurie, d'autant plus préoccupante qu'elle s'inscrit dans une tendance de fond : le problème est fondamentalement structurel.

\section{B. La recherche forestière à mobiliser}

\section{Une vaste réflexion à ouvrir}

Dans l'urgence de gérer l'après tempête, la recherche forestière s'est d'abord orientée sur le développement de techniques d'exploitation adaptées aux parcelles ravagées ainsi que sur la définition de mode de stockage des bois en attente de commercialisation. Avec l'appel d'offre lancé en avril 2000 par le GIP.ECO.FOR, le Ministère de l'Agriculture insiste aujourd'hui sur l'observation et la compréhension des conséquences de la tempête à l'échelle du paysage, du peuplement et de l'arbre ${ }^{6}$.

Au-delà du champ finalement assez étroit de l'étude d'un phénomène météorologique d'exception et de ses répercussions forestières, il nous semble qu'une réflexion de fond devrait être engagée sur la gestion des forêts françaises en profitant de l'élan de sympathie que son sort suscite actuellement parmi nos concitoyens. Comme G. Buttoud (2000), nous pensons que la tempête doit être replacée dans le cadre général de la politique forestière nationale et de son évolution afin de bien en mesurer les conséquences et de contribuer à la définition d'une "stratégie raisonnée " intégrant notamment les risques liés aux aléas naturels.

\section{La difficile étude des répercussions d'un phénomène météorologique exceptionnel}

31 La richesse des réflexions à venir sur la forêt française ne doit en tous cas pas faire oublier la difficulté intrinsèque à l'analyse des effets de la tempête sur les peuplements. Les géosystèmes forestiers sont naturellement complexes; les interactions entre facteurs humains et physiques sont nombreuses. La composition forestière, notamment en Lorraine, présente des traits spécifiques qui induisent des autocorrélations fortes entre les différentes variables explicatives, au sens statistique du terme, de la réalité sylvicole. Ainsi, les remarques sur la sensibilité particulière du Hêtre aux coups de vent renvoient à la liaison étroite entre essence dominante, type de sol et relief. Il n'est pas évident de pouvoir « débrouiller » ces interrelations.

Le problème est d'autant plus ardu que le facteur météorologique, évidemment essentiel à prendre en compte, est très difficile à cerner. L'explication fine des paysages forestiers d'après tempête, des causes de la sensibilité particulière de tel ou tel massif, risque de butter sur la faible résolution géographique des données anémométriques. Enfin, comme tout phénomène d'exception, les tempêtes de décembre 1999 portent en elle une limite à l'investigation scientifique: les forêts du sud lorrain ont été parcourues par des vents en moyenne de 120 à $160 \mathrm{~km} / \mathrm{h}$. A ce niveau de contrainte éolienne, l'étude fine de l'influence de données sylvicoles structurales, comme par 
exemple le type de traitement appliqué, a-t-elle encore un sens? N’y a-t-il pas un effet de seuil? fin 1999 se heurte avant tout à des problèmes méthodologiques. L'imprécision des premières estimations appelle à la plus grande prudence dans l'analyse et l'interprétation des données. Encore limité par l'étude de facteurs de production simples, l'examen global des conséquences de la tempête reste à développer. Malgré ces réserves, un premier bilan peut être dressé à grands traits. Exceptionnel, le phénomène météorologique du 26 décembre dernier a tout spécialement marqué les forêts lorraines. Au sein de la région, les départements de Meurthe-et-Moselle et des Vosges ont particulièrement souffert; les forêts publiques paraissent tout spécialement atteintes.

Si les événements de la fin 1999 offrent l'occasion d'une vaste réflexion sur la forêt française, celle-ci doit être recadrée à un niveau général et intégrer la dimension du temps long. La question de la gestion durable des géosystèmes forestiers demeure centrale. Elle se pose forcément à l'échelle des siècles et tend à relativiser l'impact, certes spectaculaire, mais finalement ponctuel de la tempête.

\section{BIBLIOGRAPHIE}

Badeau V. (1998). - Caractérisation écologique du réseau européen de suivi des dommages forestiers, Paris, Les cahiers du DSF, Min. Agri. Pêche, 211 p.

Buttoud G. (2000). - Politiques forestières et risques naturels, extrait de « Les écosystèmes forestiers dans la tempête », version 01 : avril 2000, Paris, GIP.ECO.FOR, pp. 17-22.

Degron R. (1999). - Forêts, Temps et Sociétés - Les conversions forestières de Lorraine : bilan, contrastes, rythmes et ruptures, Université de Nancy 2, Thèse de Doctorat en géographie, 500 p.

Doll D. (1991). - Les cataclysmes éoliens dans les forêts d'Europe, Paris, Forêt Entreprise, 5, pp. 8-9.

Husson J.P. (1991). - Les hommes et la forêt lorraine, Nancy, PUN, 318 p.

Inventaire Forestier National, 1999. Etude de la ressource forestière dans le cadre d'une réflexion stratégique sur l'utilisation du Fonds Forestier National, Lattes, IFN, non paginé.

Inventaire Forestier National, 2000. Rapport d'activité 1999, La Pâquelais, Vanden, 44 p.

Revue Géographique de l'Est, vol. 40 / 3 | 2000 
Ledoux B. (1995). - Les catastrophes naturelles en France, Paris, Payot et Rivage, 453 p.

Ministère de l'agriculture et de la pêche, 2000. La forêt française face aux tempêtes de décembre 1999. Appréciation des dégâts au niveau national à l'aide du réseau européen de suivi des dommages forestiers, Nancy, Département Santé des Forêts, 4 p.

Rameau J.C. (2000). - Ecosystème et tempête, extrait de « Les écosystèmes forestiers dans la tempête ", version 01 avril 2000, GIP.ECO.FOR, pp. 89-94.

Wencelius F. (2000). - Collecte et utilisation des données forestières - L'IFN et la tempête, actes du séminaire des 40 ans de l'IFN des 13 et 14 avril 2000 à Nancy, à paraître.

\section{NOTES}

1. Source : M. Scotto, «Les Vosges espèrent avoir rendez-vous avec le soleil » in Le Monde du $3 / 7 / 2000$.

2. Rappelons que la forêt lorraine couvre environ 841000 ha, soit un taux de boisement régional de $36 \%$ (Source : IFN, début des années 1990).

3. Dans les départements $54,55,57$ et 88 , les taux de boisement et les volumes sur pied estimés au début des années 1990 sont respectivement de : $30 \%-31 \mathrm{M} \mathrm{m3}, 37 \%-36 \mathrm{M} \mathrm{m3}, 27 \%-40 \mathrm{M}$ $\mathrm{m} 3$ et $48 \%-70 \mathrm{M} \mathrm{m} 3$

4. En Moselle, les forêts domaniales représentent les deux tiers des forêts publiques. Dans le reste de la région, elles n'en représentent que le tiers.

5. Sources: ONF Lorraine, Six mois après la tempête, juin 2000 et C.R.P.F. Lorraine-Alsace, compte-rendu de la tournée "Reconstitution » en Meuse et en Meurthe-et-Moselle du 20 avril 2000

6. Source : Appel à propositions - Recherches forestières à conduire à court terme suite aux tempêtes de fin décembre 1999, GIP.ECO. FOR, avril 2000.

\section{RÉSUMÉS}

L'étude des conséquences forestières des tempêtes de la fin 1999 pose d'importants problèmes méthodologiques. Malgré l'imprécision des données aujourd'hui à disposition, un premier bilan des volumes de bois tombés et des surfaces forestières touchées en Lorraine révèle la sensibilité particulière de la région et certains contrastes entre départements et types de propriétaire. Une vaste réflexion paraît devoir être engagée sur le thème du développement durable des géosystèmes forestiers.

Study consequences for forests of the storms at the end of 1999 raises some important methodological problems. Despite the imprecision of data we have today, a first assessment of the volume of faller timber and forest areas affected in Lorraine shows a particular regional sensitivity and some contrasts between "départements " and types of owner. A substantial period of reflection seems to be starting on the topic of the sustainable development of forest geosystems. 
Die Untersuchung der forstlichen Auswirkungen der Stürme Ende 1999 stellt wichtige methodologische Probleme. Trotz der Ungenauigkeit der heute verfügbaren Daten lässt eine erste Bilanz über die Menge des gefallenen Holzes und die in Lothringen betroffene forstliche Fläche die besondere Anfälligkeit der Region und gewisse Gegensätze zwischen den Besitzrevieren und typen erkennen. Eine eingehende Überlegung zum Thema der nachhaltigen Entwicklung der forstlichen Geosysteme scheint in Gang kommen zu müssen.

\section{INDEX}

Keywords : forest, forest inventory, Lorraine, sustainable development, tempest Mots-clés : développement durable, forêt, inventaire forestier, Lorraine, tempête

Schlüsselwörter : Forst, Forstinventar, Lothringen, Nachhaltige Entwicklung, Sturm

\section{AUTEUR}

\section{ROBIN DEGRON}

Direction Régionale de l'Environnement de Lorraine - 19, avenue Foch, 57000 Metz 